\title{
Study on the Expression of PCNA in Hypertrophic Scar Cell Model
}

\author{
Rong'an $\mathrm{Hao}^{1,2}$, Yu An ${ }^{1,2}$, Kun Yuan ${ }^{2}$
}

\author{
${ }^{1}$ Department of Medicine, Qingdao University, Shandong, Qingdao, 266003 \\ ${ }^{2}$ Department of Medical Cosmetic Center, Affiliated Hospital of Qingdao University, Shandong, Qingdao, 266003
}

\begin{abstract}
Objective Study the expression of PCNA under different mechanical tension on the transformation of normal skin fibroblasts (NSFB) to hypertrophic scar fibroblasts(HSFB). Methods NSFB and HSFB were divided into the stretch group and the control group. Stretch groups were loaded with $0.1 \mathrm{~Hz}, 10 \%$ amplitude cyclic stretch for 3, 5 and 7 days respectively. Control group were cultured without cyclic stretch. At the end of experiments, cell proliferation was determined by CCK8, immunocytochemistry (ICC) of proliferating cell nuclear antigen (PCNA). Results When loaded for 5 days with amplitude cyclic stretch, compared with HSFB control group, expression levels of PCNA of the NSFB stretch group were no significant differences $(\mathrm{P}>0.05)$; while loaded for 3 days or 7 days, the above indexes showed significant differences $(\mathrm{P}<0.05)$. Conclusion The effect of mechanical tension for 5 days is the best time, which can induce normal skin fibroblasts to show some biochemical characteristics of hypertrophic scar fibroblasts.
\end{abstract}

\section{Introduction}

Wound healing can also lead to scar formation, resulting in uneven, irregular shape, local flushing and hypertrophic scar with burning pain and pruritus.In addition, when hypertrophic scar occurs on the face, joints and hands, it will lead to the generation of contracture, serious functional disorder, aesthetic effect and huge physiological and psychological burden for patients, which is one of the urgent problems to be solved in orthopaedics and burn department[1]. Hypertrophic scars often occur at the depth of damage involving dermal trauma. Compared with normal scars, fibroblasts in the formation process are more active, and collagen deposition is more obvious, leading to more pronounced dermal fibrosis. Early research has shown that the skin fibroblasts of $10 \%$ tensile amplitude cyclic mechanical tension[2] of the mechanical transmission effect and biochemical reaction, and strength of fibroblasts have showed the trend of HSFB some phenotypes, such as the TGF beta 1, the expression of collagen type I and alpha SMA.However, the cell proliferation of NSFB transformed into HSFB under mechanical tension at different time is not clear. In this study, mechanical tension was used to induce NSFB to HSFB transformation, to observe the effect of NSFB to HSFB transformation on cell proliferation under mechanical tension for a certain period of time, and to explore the optimal loading time of mechanical tension to induce human NSFB to HSFB transformation, laying a foundation for the study of hypertrophic scar formation mechanism.

\section{Materials and Methods}

\subsection{Main reagents and Instruments}

$\mathrm{CO} 2$ culture incubator (Panasonic, Japan ) , super-clean working table (AIRTech, Japan), inverted microscope (Olympus, Japan), 6-well elastic substrate culture plate ( 6-well BioFlex ${ }^{\circledR}$ culture plate )

(BF-3001C, Flex-cell, USA), 6-well culture plate (COSTASR, USA), multi-channel cells stress loading instrument (Flex-cell, USA), Chemiluminescence imaging system (ALPHA FCE, USA) , $4^{\circ} \mathrm{C} 、-20^{\circ} \mathrm{C}$ refrigerator (Haier, Qingdao, China), $-80^{\circ} \mathrm{C}$ refrigerator (Sanyo, Japan), high speed centrifuge (Thermo Fisher, USA), $4^{\circ} \mathrm{C}$ centrifuge (Thermo Fisher, USA), micropipette (Eppendorf, German).

Fetal Bovine Serum, Penicillin-Streptomycin, EDTAtrypsin (Gibco, USA) , MEM Eagle (CORNING, USA ) , phosphate buffer solution ( PBS ), dimethylsulfoxide (DMSO) (Solarbio, Beijing, China), Cell Counting Kit-8 (CCK-8) (DOJINDO, Japan ) , TRIzol Reagent (Ambion, USA ) , trichloromethane, isopropanol, absolute ethyl alcohol

(Haoran, Shanghai, China), Reverse transcription kit (RR047Q), Amplification kit (RR820A) (Takara, Dalian, China).

\subsection{Cell culture and Grouping}

The scar tissue and normal dermal tissue used in the experiment were stored in a refrigerator in the medical research center of Qianfoshan hospital in Jinan, Shandong province. The experiment is in compliance with the requirements of the Research Ethics Committee of Qingdao university and passed the examination. None of the patients had other basic 
diseases, and all the scars showed hyperplasia.The scarring of red or dusky-red and hard texture was more than $0.5 \mathrm{~cm}$ above the normal surrounding skin surface, and no antitumor drugs, hormone drugs or radiotherapy were performed before surgery.

Fibroblasts were cultured in vitro according to the literature using tissue mass method, and the cells of the third generation to the eighth generation were selected for the experiment.NSFB and HSFB were divided into experimental group and control group. According to the instructions of the planimeter, the cells in the experimental group were suspended for 1 hour after 4 hours of continuous planishing, and the cycle lasted no less than 10 hours per day. The total time for loading mechanical tension was divided into 3 days group, 5 days group and 7 days group.Strength environment is still the $37^{\circ} \mathrm{C}, 5 \% \mathrm{CO} 2$ and saturated humidity.In the control group, no force was applied, and the other culture conditions were the same as those in the experimental group.Each group was repeated three times. The cells of the experimental group and the control group were supplemented with $0.5 \mathrm{ml}$ serum culture solution of $10 \%$ per well every two days.

\subsection{Immunocytochemistry（ICC）}

For ICC, expression of PCNA is widely used as a marker of replicated DNA[4] and cell proliferation[5]. Control Cells were seeds in the normal 6-well plates with polylysine-coated slides. At each testing time point, experimental group cells in the 6-well elastic substrate culture plates were transferred to the normal 6-well plates with polylysine-coated slides by trypsinization overnight. Cells were washed three times with PBS, then fixed with $4 \%$ paraformaldehyde for 15 minutes and permeabilized with $0.3 \%$ Triton X-100 for 10 minutes at room temperature (RT). After blocked with $5 \%$ bovine serum albumin (BSA) for $1 \mathrm{~h}$ at RT, the cells were treated with a mixture of $\mathrm{H} 2 \mathrm{O} 2$ and methanol, at a ratio of $1: 40$, to block the endogenous peroxidase activity. Subsequently, cells were incubation with 1: 200 diluted primary PCNA antibody

(bs-2006R, BIOSS) overnight at $4^{\circ} \mathrm{C}$. Thereafter, the samples were incubated with Peroxidase-Conjugated Goat anti-Rabbit $\operatorname{IgG}(\mathrm{H}+\mathrm{L})$ as secondary antibody

(ZB-2301, ZSGB-BIO, Beijing) for $1 \mathrm{~h}$ at RT and were visualized with metal-enhanced 393-diaminobenzidine (DAB)(Boster, Wuhan, China) for $5 \mathrm{~min}$ (ABC-DAB method).To visualize a clearer structure, we stained them with hematoxylin for $15 \mathrm{~s}$ and then dehydrated them in a graded series of alcohols and xylene.Finally, the specimens were photographed under a light microscope or a confocal laser scanning microscope (FV10-ASW2.1 Viewer).

\subsection{Cell proliferation assays by $\mathrm{CCK}-8$}

NSFB and FSFB proliferation were determined using a
Cell Counting kit (CCK-8) . 3 wells of 6-well plates in each group used in CCK-8 and the other 3 wells for ICC.For the CCK-8 assay, at each testing time point, $200 \mu \mathrm{L}$ of sterile CCK-8 solution was added to each well by well mixing and incubated for $3 \mathrm{~h}$ at $37^{\circ} \mathrm{C}$. At the end of the incubation, $200 \mu \mathrm{L} /$ well cell culture supernatant was added in 96-well plates, meanwhile, Culture medium without cells was used as control. Then, microplate reader was used to determine the absorbance at $450 \mathrm{~nm}$ according to the manufacturer's instructions.

\subsection{Statistical analysis}

The results are presented as mean \pm standard deviation (SD). Treatment effects were compared using one-way ANOVA among different groups. All analyses were performed using GraphPad Prism Version 5.0 (San Diego, USA). $\mathrm{P}<0.05$ was considered statistically significant.

\section{Results}

2.1 Proliferation of cells under different stress time The proliferation of HSFB was higher than that of NSFB.The proliferation capacity of cells in the experimental group increased with the increase of stress time.After 3 and 7 days, the proliferation of the HSFB experimental group was higher than that of the HSFB control group, the proliferation of the NSFB experimental group was higher than that of the NSFB control group, and the proliferation of HSFB was higher than that of the NSFB control group.The proliferation capacity of NSFB experimental group was the same as that of HSFB (P > 0.05). (Fig.1)

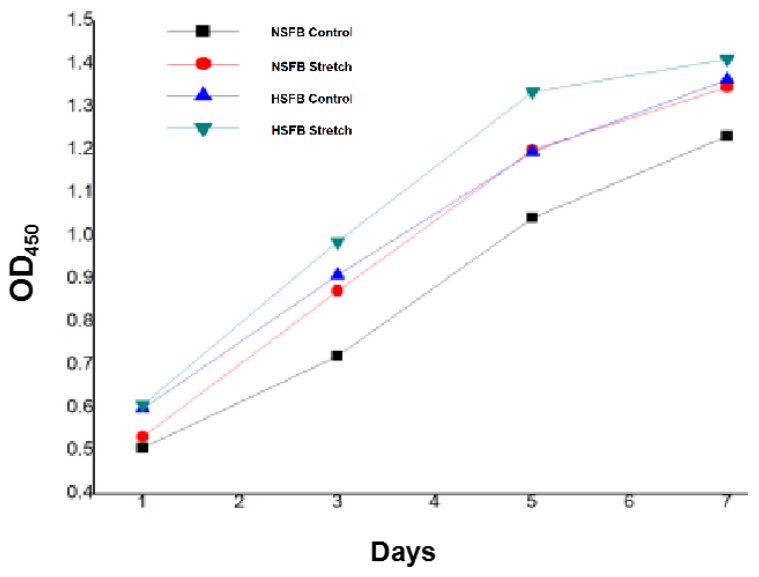

Fig.1 The proliferation capacity of cells in each group was detected by cck- 8 method

2.2 Expressions of PCNA under different stress time The micrograph showed that the PCNA expression in NSFB stretch group within 5days was basically consistent with that of HSFB control group. (Fig.2 $5 b-5 c)$ 


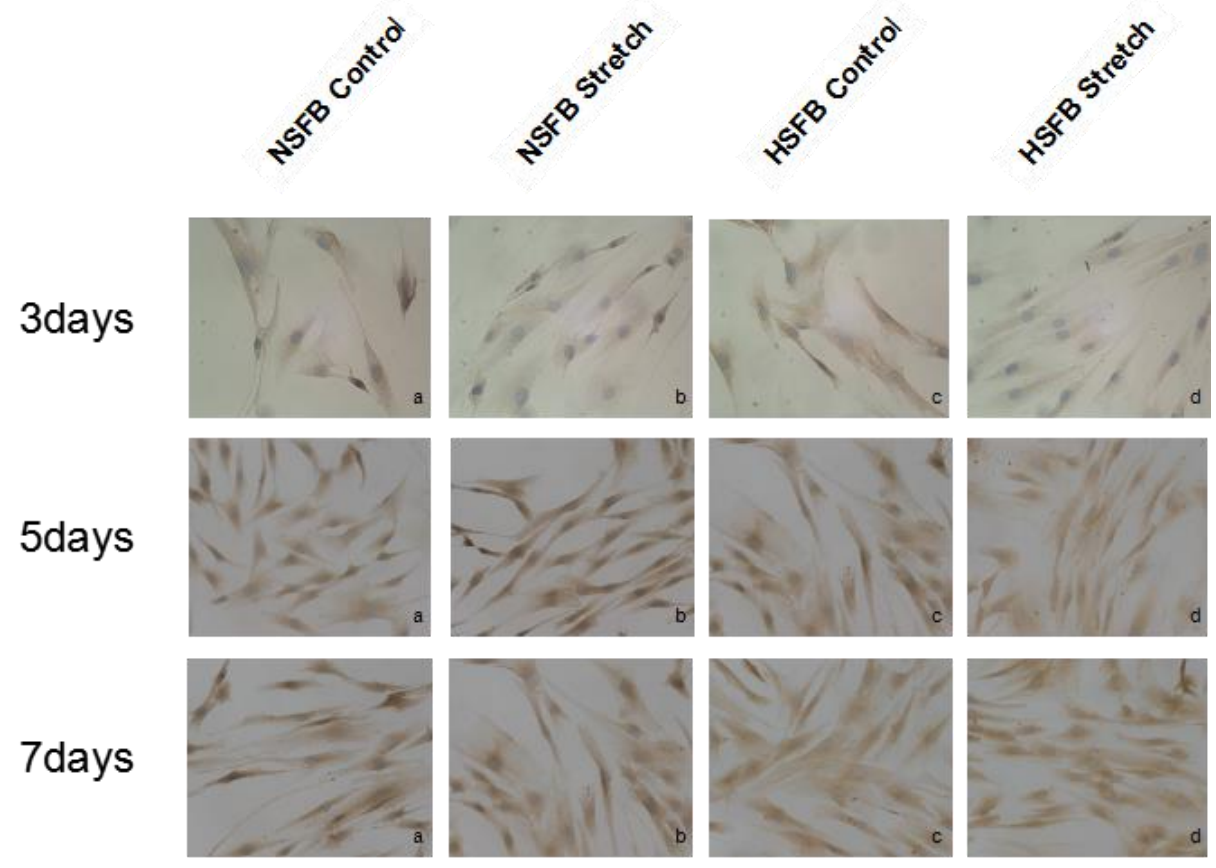

Fig.2 The expression of PCNA in the stretch group (brown yellow) showed an increasing tendency to compare with the ICC staining in the control group (original magnification x 200).

\section{Discussion}

PCNA is an evolutionarily highly conserved protein with a relative molecular mass of $36 \mathrm{ku}$.Its main function is to identify the primal-template complex together with the replicator $\mathrm{C}$ as the auxiliary protein of DNA polymerase protein[3], so as to help the polymerase protein to locate, promote its DNA extension activity, and ensure that it will not fall off the DNA strand during the replication process, so as to directly participate in the DNA replication process of cell proliferation[4]. Secondly, PCNA is also a kind of cell cycle regulation protein existing in the nucleus, interacting with cell cycle protein, cell cycle protein-dependent kinase and cell cycle protein-dependent kinase inhibitory protein to regulate the transformation of each phase of cell cycle. As PCNA only appears in cells with proliferation state, and the expression level reaches the peak in the $S$ phase of cell proliferation, it is taken as an important indicator to evaluate the cell proliferation state[5].

Fibroblast activity plays an important role in the development of hypertrophic scars. The active functions of fibroblasts include proliferation and differentiation, synthesis and secretion of extracellular matrix.From the available literature, we have not found a method to determine the proliferation activity of fibroblasts in scar tissue.

Extensive defects of skin tissue are often caused by severe burns, trauma, intractable skin ulcers of diabetes and tumor resection[6]. Normal healing of defect skin is the result of the balance of fibroblasts, cytokines and extracellular matrix. When the imbalance of cytokines, extracellular matrix and fibroblasts is regulated, it may lead to changes in the biological behavior of fibroblasts and form hypertrophic scars.Hyperplastic scar tissue fibroblasts within a large number of aggregation, collagen type I and III ratio anomaly, collagen content increasing, affect beautiful, even lead to severe deformity and dysfunction, reduce the patients quality of life.

The present study suggests that proliferative exhausted marks although complex formation, but can determine fibroblasts is an important participant, the abnormal cells of some key genes, result in changes of certain signaling pathways, and the abnormal signals, to guide the cell cycle change leads to a lot of good breeding, normal cell death of cells decreased, these changes may be raised the main lure for organic scarring.

On the molecular level, abnormal proliferation and differentiation of fibroblasts play a key role in hypertrophic scar formation and remodeling[7].Therefore, in this study, we evaluated the expression of PCNA, a DNA polymerase delta helper protein that is synthesized and expressed in proliferative cells and is critical for cell replication and cell cycle progression[8].Our current data show that NSFB has the same value-added state as HSFB after 5 days of exertion. The time window for treatment and inhibition of hypertrophic scar formation was further confirmed.As for the differences in PCNA protein expression in this study, we need to further study and carefully control each time point to confirm. However, 
gene proteins and mRNA expression are not always parallel because they are regulated differently.

\section{Conclusion}

In conclusion, the experimental results indicated that the PCNA expression of HSFB was significantly higher than that of normal skin fibroblasts, but there were differences in the expression of both. The two types of cells were in different proliferation states, and the PCNA expression was similar only in the case of 5 days of stress.

\section{Conflict of interest}

We wish to confirm that there are no known conflicts of interest associated with this publication and there has been no significant financial support for this work that could have influenced its outcome.

\section{Acknowledgments}

Rong'An was responsible for experimental design and performed research, analysis, and interpretation of the results; drafted the manuscript; and provided final approval of the version to be published. An YU and Kun Yuan participated in the design of this study and provided general support. All authors helped perform the analysis with constructive discussions. All authors read and approved the final manuscript.

\section{References}

1. Su, S., S. Xin, and Y. Yu, [The study of expression and distribution of PCNA in hypertrophic scar tissue]. Zhonghua zheng xing wai ke za zhi $=$ Zhonghua zhengxing waike zazhi $=$ Chinese journal of plastic surgery, 2000. 16(2): p. 73-74.

2. Junker, J.P.E., et al., Mechanical tension stimulates the transdifferentiation of fibroblasts into myofibroblasts in human burn scars. Burns, 2008. 34(7): p. 942-946.

3. Hunt, B.G., et al., Maternal diethylhexyl phthalate exposure affects adiposity and insulin tolerance in offspring in a PCNA-dependent manner. Environmental Research, 2017. 159: p. 588-594.

4. Hilton, B.A., et al., Progerin sequestration of PCNA promotes replication fork collapse and mislocalization of XPA in laminopathy-related progeroid syndromes. The FASEB Journal, 2017. 31(9): p. 3882-3893.

5. Dezfuli, B.S., et al., Liver of the fish Gymnotus inaequilabiatus and nematode larvae infection: Histochemical features and expression of proliferative cell nuclear antigen. Journal of Fish Diseases, 2017. 40(12): p. 1765-1774.

6. Derderian, C.A., et al., Mechanical Strain Alters Gene Expression in an in Vitro Model of Hypertrophic Scarring. Annals of Plastic Surgery, 2005. 55(1): p. 69-75.

7. Sun, T., et al., [The characteristics of PCNA expression in hypertrophic scars and chronic ulcers and implication of the expression]. Zhonghua zheng xing wai ke za zhi = Zhonghua zhengxing waike zazhi $=$ Chinese journal of plastic surgery, 2002. 18(4): p. 232-233.

8. Zhao, B.C. and L. Qian, [Detection of apoptosis by in situ labeling and study on the expression of PCNA in hypertrophic scars and keloids]. Hunan yi ke da xue xue bao = Hunan yike daxue xuebao $=$ Bulletin of Hunan Medical University, 2000. 25(1): p. 73-76. 